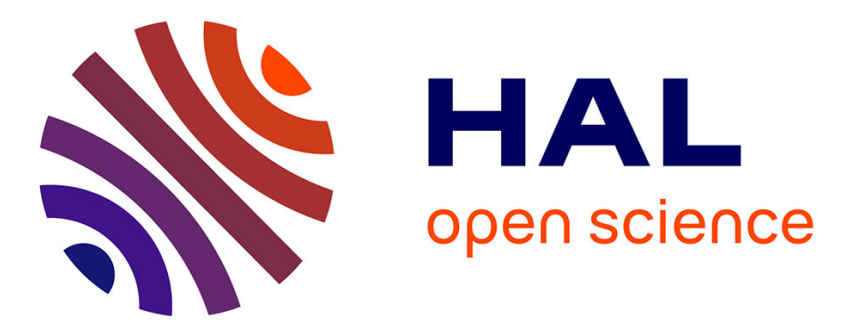

\title{
High-energy emission from a magnetar giant flare in the Sculptor galaxy
}

\author{
M. Ajello, W.B. Atwood, M. Axelsson, L. Baldini, G. Barbiellini, M.G. \\ Baring, D. Bastieri, R. Bellazzini, A. Berretta, E. Bissaldi, et al.
}

\section{- To cite this version:}

M. Ajello, W.B. Atwood, M. Axelsson, L. Baldini, G. Barbiellini, et al.. High-energy emission from a magnetar giant flare in the Sculptor galaxy. Nature Astron., 2021, 5 (4), pp.385-391. 10.1038/s41550020-01287-8 . hal-03210381

\section{HAL Id: hal-03210381 \\ https://hal.science/hal-03210381}

Submitted on 29 Apr 2021

HAL is a multi-disciplinary open access archive for the deposit and dissemination of scientific research documents, whether they are published or not. The documents may come from teaching and research institutions in France or abroad, or from public or private research centers.
L'archive ouverte pluridisciplinaire HAL, est destinée au dépôt et à la diffusion de documents scientifiques de niveau recherche, publiés ou non, émanant des établissements d'enseignement et de recherche français ou étrangers, des laboratoires publics ou privés. 


\title{
High-Energy Emission from a Magnetar Giant Flare in the Sculptor Galaxy
}

\author{
3 The Fermi LAT collaboration*
}

\section{ABSTRACT}

Magnetars are the most highly-magnetized neutron stars in the cosmos $\left(B \sim 10^{13-15} \mathbf{G}\right)$. Giant flares from magnetars are rare, short-duration (about $0.1 \mathrm{~s}$ ) bursts of hard X-rays and soft $\gamma$ rays ${ }^{1,2}$. We report here the discovery of GeV emission from a magnetar giant flare (MGF) on 15 April, $2020^{3-5}$. The Large Area Telescope (LAT) on board the Fermi Gamma-ray Space Telescope detected GeV $\gamma$ rays from $19 \mathrm{~s}$ until $284 \mathrm{~s}$ after the initial detection of a signal in the $\mathrm{MeV}$ band. Our analysis shows that these $\gamma$ rays are spatially associated with the nearby $(3.5 \mathrm{Mpc})$ Sculptor galaxy and are unlikely to originate from a cosmological $\gamma$-ray burst. Thus, we infer that the $\gamma$ rays originated with the MGF in Sculptor. We suggest that the GeV signal is generated by an ultra-relativistic outflow that first radiates the prompt MeV-band photons, and then deposits its energy far from the stellar magnetosphere. After a propagation delay, the outflow interacts with environmental gas, produces shock waves that accelerate electrons to very high energies and these then emit $\mathrm{GeV} \gamma$ rays as optically thin synchrotron radiation.

On 15 Apr 2020, the Fermi Gamma-ray Burst Monitor (GBM) triggered and located GRB 200415A ${ }^{4}$ initially classified as a short (duration $<2$ seconds) Gamma-ray Burst (SGRB). The Interplanetary Network of $\gamma$-ray detectors (IPN ${ }^{1}$ ) reduced the uncertainty on the GBM position to $20 \mathrm{sq}$. arcmin suggesting that the GRB originated from the nearby Sculptor galaxy $^{3}$, located at a distance of $\sim 3.5 \mathrm{Mpc}^{6}$. This, with the resemblance of the GBM sub-MeV light curve ${ }^{7}$ to the extragalactic Soft Gamma Repeater (SGR) giant flare candidates GRB $051103^{3,8}$ and GRB $070201^{9}$, and the detection of quasi-periodic oscillations (QPOs) by the Atmosphere-Space Interaction Monitor (ASIM) ${ }^{5}$, led to the identification of GRB 200415A as a Magnetar Giant Flare (MGF) in Sculptor. GRB 200415A was $43^{\circ}$ from the LAT boresight at the GBM trigger time $\mathrm{T}_{0}$ (08:48:05.563746 UTC) and remained well within the LAT field of view (FOV) until 500 seconds after $\mathrm{T}_{0}$. Three $\gamma$ rays were detected by the LAT, allowing the localization of GRB 200415A at high energies ( $>100 \mathrm{MeV})$ : this represents the first detection of high-energy gamma-ray emission from an MGF, and suggests that magnetars can power the relativistic outflows observed in some SGRBs.

To study the localization of the $\gamma$-ray signal observed by the LAT we perform a likelihood analysis and compute a test statistic (TS) for the presence of the source at different positions. The best position is obtained from the maximum of the $\mathrm{TS}\left(\mathrm{TS}_{\max }=29\right.$, corresponding to a detection significance close to $5 \sigma$, see the Method section). Then, the variation of the TS around this position provides the map of localization contours shown in Figure 1. The iso-contours in red encompass localization probabilities of $68 \%$ and $90 \%$. Four galaxies (IC 1576, IC 1578, IC 1582 and NGC 253) from the NGC 2000 catalog ${ }^{10}$ are located within a circular region of radius $r_{99}$, whose area is equivalent to the $99 \%$ c.l., and which is centered on the maximum of the TS map at R.A., Dec. $=11.13^{\circ},-24.97^{\circ}(\mathrm{J} 2000)$. NGC 253, also known as the Sculptor galaxy, with its high star-formation activity and flux integrated between $100 \mathrm{MeV}$ and $100 \mathrm{GeV}$ of $(1.3 \pm 0.2) \times 10^{-8} \mathrm{~cm}^{-2} \mathrm{~s}^{-1}$, is a starburst galaxy already detected in $\gamma$ rays ${ }^{11,12}$. The center of the galaxy lies on the contour containing a localization probability of $72 \%$.

We apply the likelihood ratio (LR) $\operatorname{method}^{13}$ to quantify the reliability of a possible association of the $\gamma$-ray source with Sculptor. This method can distinguish between two situations: the true counterpart associated with a $\gamma$-ray emitter, which appears to lie at a certain distance due to localization uncertainties, or a background object which, by chance, happens to lie close to the $\gamma$-ray position. Our analysis takes into account the angular size of the counterpart candidate and the elongated shape of the LAT localization contours shown in Figure 1. Since the LR method takes into account the magnitude of the galaxy, we find that the Sculptor galaxy is the most likely host galaxy of the source detected by the LAT with a LR value $\sim 60$ times larger than the values for other galaxies. To evaluate the statistical significance of this association, we compare the LR values obtained in these analyses with the same analyses repeated over a sample of random locations in the sky. The p-values range from $3.2 \times 10^{-4}$ to $2.9 \times 10^{-3}$ depending on the particular analysis (see details in the Method section). Both analyses suggest positional association between Sculptor and the LAT $\gamma$-ray detection. Assuming that the emission detected by the LAT is from an SGRB, our calculation of the False Alarm Rates (FARs) range from $5.4 \times 10^{-4} \mathrm{yr}^{-1}$ to $4.7 \times 10^{-3} \mathrm{yr}^{-1}$.

We perform a detailed maximum likelihood spectral analysis of the LAT emission by modeling GRB 200415A as a point source with a power-law spectrum. As part of our analysis we estimate the probability that each photon detected by the LAT is associated with the point source, as opposed to any of the other model components. Three events are associated with the

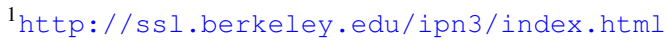




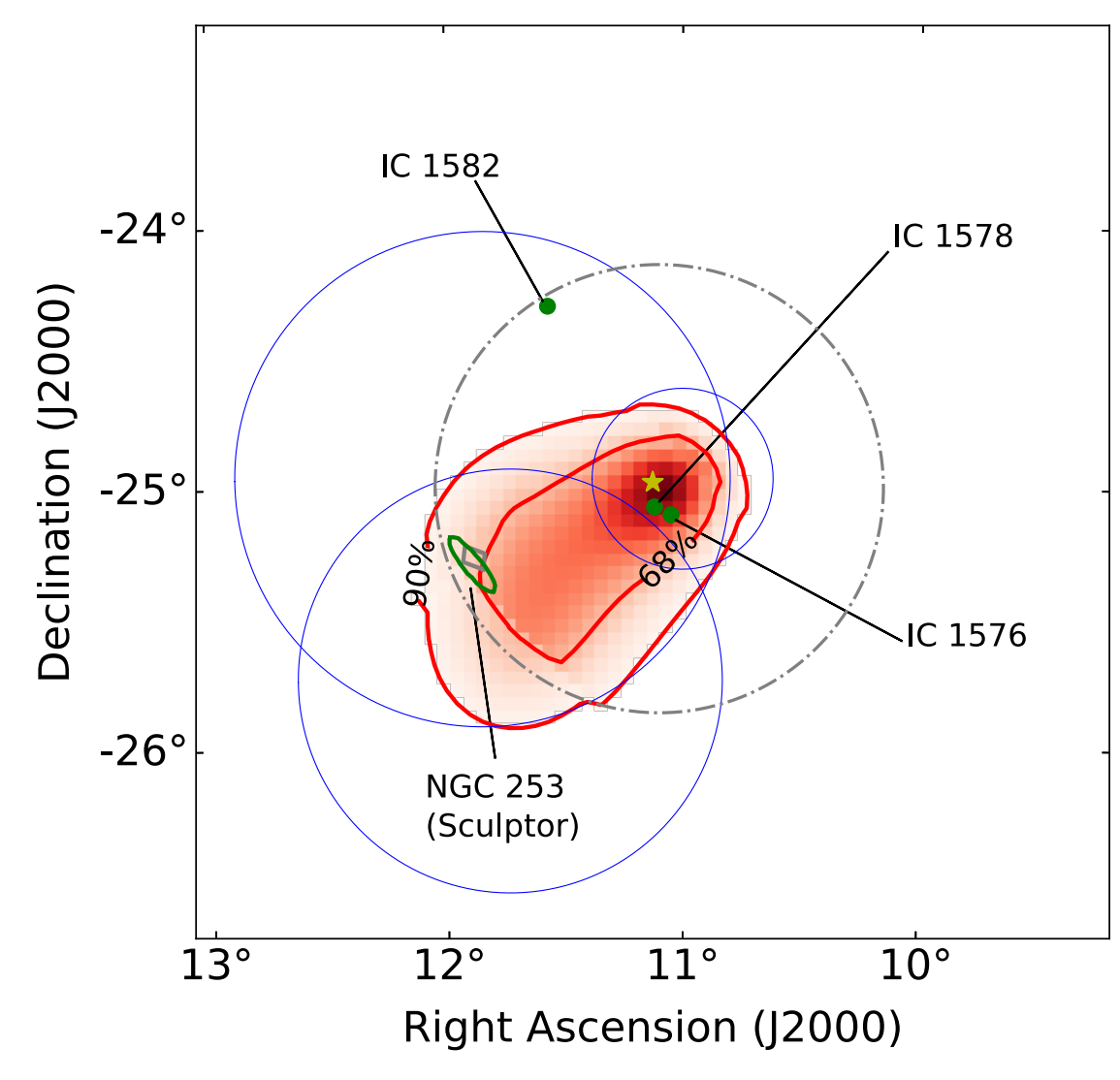

80

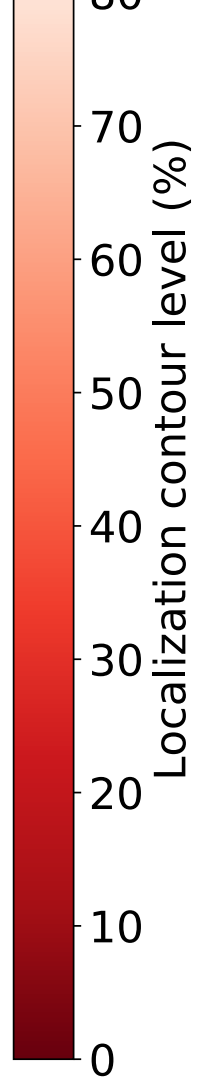

Figure 1. Map of the localization contour probability. The contours encompassing a probability of $68 \%$ and $90 \%$ are displayed in red, while the yellow star marks the location of the TS maximum. Galaxies from the NGC 2000 catalog are shown as green disks, except NGC 253 (Sculptor galaxy), which is shown as an extended source. The gray box indicates the localization provided by the IPN ${ }^{3}$. The circle whose area is equivalent to the $99 \%$ confidence level is displayed with a gray dashed-dot line, while the blue circles indicate the $68 \%$ containment of the PSF for the three $\gamma$ rays likely associated with the flare.

source with a probability greater than $90 \%$. The arrival times (after $\mathrm{T}_{0}$ ) of these events are 19, 180 and 284 seconds; with energies $480 \mathrm{MeV}, 1.3 \mathrm{GeV}$ and $1.7 \mathrm{GeV}$; respectively. The reconstructed directions of these events are shown in Figure 1 as circles with a radius equal to the point-spread function (PSF) of the instrument at their respective energies. To estimate the significance of this cluster of three events (triplet) and the probability that it is due to a background fluctuation, we look at a region of $1^{\circ}$ radius around the location of Sculptor using the entire LAT data set available (more than 12 years of data). Two different analyses, applying $\mathrm{Li} \& \mathrm{Ma}^{14}$ and Bayesian Blocks (BB) methods ${ }^{15}, 16$, result respectively in p-values of $\mathrm{p}_{\mathrm{Li} \& \mathrm{Ma}}=$ $8.3 \times 10^{-7}$ and $\mathrm{p}_{\mathrm{BB}}=2.3 \times 10^{-3}$. Finally, we calculate the rate of chance coincidence between a LAT triplet signal and a GBM SGRB in the same region of Sculptor within a given time window. The FARs for the two analyses are $1.6 \times 10^{-7} \mathrm{yr}^{-1}$ and $6.3 \times 10^{-8} \mathrm{yr}^{-1}$ respectively. 
To summarize, the FAR to detect high-energy emission from an SGRB spatially associated by chance to Sculptor is one event in $\sim 200-1800$ years, depending on the analysis method, while the FAR to also have the event temporally coincident with a GBM SGRB is of the order of 1 every $\sim 10^{6}-10^{7}$ years. Accordingly, we conclude that the LAT signal is associated with an MGF event in Sculptor. This represents the first detection of $>100 \mathrm{MeV} \gamma$ rays from any magnetar (Galactic or extragalactic).

The intense GBM emission below $1 \mathrm{MeV}$ defines the so-called "initial spike" of the MGF and must come from a relativistic wind $^{4}$. The three local magnetars that have displayed MGFS (two in the Milky Way and one in the Large Magellanic Cloud) each had pulsating late-time emission of effective temperature $10-25 \mathrm{keV}$, emitting $\sim 10^{44}$ erg of energy over a few hundred seconds. The LAT signal cannot come from this region $\left(R \lesssim 3 \times 10^{7} \mathrm{~cm}\right)$ due to the high opacity ${ }^{17}$ to $\gamma \rightarrow e^{+} e^{-}$pair creation in the magnetar's enormous magnetic field. The long $\left(t_{\mathrm{del}}=19 \mathrm{~s}\right)$ delay between the initial spike and first LAT photon detection suggests that the $\mathrm{GeV}$ emission must take place well outside the light cylinder radius $P c / 2 \pi \sim 10^{10}-10^{11} \mathrm{~cm}$ for magnetars of rotation periods $P \sim 2-12 \mathrm{~s}$. Thus, the scenario we propose is that the GeV emission arises from dissipation associated with the collision between an ultra-relativistic outflow from the MGF and an external shell of swept-up material. The huge energy release, $\sim 10^{47} \mathrm{erg}$, within $\sim 0.14 \mathrm{~s}^{4}$ likely from magnetically-induced crustal fracturing of the magnetar surface ${ }^{18}$ or from the deformation of the magnetosphere ${ }^{19,20}$ creates a very hot plasma. Initially the radiation is trapped inside this magnetized plasma rich in electron-positron pairs and vastly fewer baryons. The plasma accelerates under its own radiation pressure and becomes optically transparent to electron scattering at distances $R>10^{8} \mathrm{~cm}$ from the magnetar. The emission of radiation from a range of radii and with a range of effective temperatures $\lesssim 300 \mathrm{keV}$ constitutes a Comptonized spectrum peaking at $\sim 1$ $\mathrm{MeV}$, as observed by the GBM. The accompanying plasma continues its outward flow with a bulk Lorentz factor $\Gamma_{\text {ej }} \sim 100$ and kinetic energy $\sim 3 \times 10^{46} \mathrm{erg}^{21,22}$. Such a high Lotentz factor is in contrast to the MGFs observed in the Milky Way that powered only mildly relativistic outflows observed as radio nebulae ${ }^{23,24}$ expanding at $\sim .7 \mathrm{c}$, where the much lower expansion velocity can be attributed to entrainment of a larger baryon mass. The inferred kinetic energy of the outflow from the MGF in Sculptor is, however, comparable with the total radiated energy in the initial spike, as also inferred for the previous local MGFs.

In its quiescent state, the magnetar putatively emits a pulsar-type ultra-relativistic Magnetohydrodynamics wind powered by its spin-down energy. The continual wind sweeps up interstellar gas, and stalls at a bow shock forming a shell at a distance $R_{\mathrm{bs}} \sim 8 \times 10^{15} \mathrm{~cm}$. The MGF outflow, which itself becomes a thin shell over time, therefore propagates essentially in an evacuated cavity until it collides with the bow-shock shell. The time of collision is $\approx R_{\mathrm{bs}} / 2 \Gamma_{\mathrm{ej}}^{2} c \sim 10 \mathrm{~s}$ which is similar to the time $t_{\mathrm{del}}$. After collision, a forward shock propagates in the bow-shock shell and a reverse shock propagates in the MGF shell. Electrons are accelerated at the shocks to relativistic energies and emit synchrotron radiation up to GeV energies in shock-generated magnetic fields. The duration of the peak emission is $\approx R_{\mathrm{bs}} / 2 \Gamma_{\mathrm{sh}}^{2} c \sim 400 \mathrm{~s}$, where $\Gamma_{\mathrm{sh}} \sim 20$ is the bulk Lorentz factor of the forward shock. This is the time scale over which the LAT-detected synchrotron photons with energies up to a few $\mathrm{GeV}$ (see the Methods section for details).

GRB 200415A is the first case of an MGF detected at $\gtrsim 100 \mathrm{MeV}$ energies, noting that similarities between the MGFs and cosmological GRBs have been pointed out in the past ${ }^{2,25,26}$. Previous searches in LAT data for persistent hard $\gamma$-ray emission from several Galactic magnetars resulted in stringent upper limits ${ }^{27,28}$. The $10-500$ seconds (from $\mathrm{T}_{0}$ ) LAT spectrum of GRB 200415A, with a photon index $\Gamma=-1.7 \pm 0.3$ and a flux of $(4.1 \pm 2.2) \times 10^{-6} \mathrm{~cm}^{-2} \mathrm{~s}^{-1}$ (two orders of magnitude brighter than the non-variable flux of Sculptor), is typical of an SGRB detected by the LAT. What makes GRB 200415A different from other LAT-detected SGRBs is the long delay, $\sim 19$ s compared to $\lesssim 1$ s typical values, between the GBM trigger time and the LAT detection ${ }^{29}$ (see the Method section). Among the 17 SGRBs detected by the LAT in the first 10 years, GRB 200415A shows the longest delay between the end of the GBM-detected emission and the beginning of the high-energy emission, and only two SGRBs were detected by the LAT for a duration comparable to that of GRB 200415A. While these peculiarities by themselves do not rule out GRB 200415A being a cosmological SGRB, its association with Sculptor, its very flat GBM spectrum below $1 \mathrm{MeV}^{4}$, and the quasi-periodic oscillation (QPO) detection by $\mathrm{ASIM}^{5}$, strongly point toward an MGF origin.

We suggest that an ultra-relativistic outflow with energy similar to the prompt $\gamma$-ray energy emanated from the MGF in Sculptor and it hit a dense shell of material surrounding the magnetar. Shock-heated material accelerated electrons to relativistic energies which emitted synchrotron radiation in the presence of a magnetic field generated in the shocks. The LAT detected the high-energy component of the spectrum for the first time from an MGF as GRB 200415A.

\section{References}

1. Frail, D. A., Kulkarni, S. R. \& Bloom, J. S. An outburst of relativistic particles from the soft $\gamma$-ray repeater SGR1900+14. Nature 398, 127-129, DOI: 10.1038/18163 (1999). astro-ph/9812457.

2. Hurley, K. et al. An exceptionally bright flare from SGR 1806-20 and the origins of short-duration $\gamma$-ray bursts. Nature 434, 1098-1103, DOI: 10.1038/nature03519 (2005). astro-ph/0502329. 
3. Svinkin, D. \& the IPN Team. Bright twin $\gamma$-ray flares in two nearby galaxies as giant magnetar flares. Submitted to Nature (2020).

4. Roberts, O. J. \& the GBM and Swift Teams. Rapid Spectral Variability of a Giant Flare from an Extragalactic Magnetar. Submitted to Nature (2020).

5. Castro-Tirado, A. J. \& the ASIM Team. Discovery of quasi-periodic oscillations in the complex light curve from a giant flare from a distant magnetar. Submitted to Nature (2020).

6. Rekola, R. et al. Distance to NGC 253 based on the planetary nebula luminosity function. Mon. Notices Royal Astron. Soc. 361, 330-336, DOI: 10.1111/j.1365-2966.2005.09166.x (2005). https://academic.oup.com/mnras/article-pdf/361/1/330/ 18658607/361-1-330.pdf.

7. Burns, E. Extragalactic Magnetar Giant Flares are a Class of Gamma-Ray Bursts. Submitted to Nature (2020).

8. Frederiks, D. D. et al. On the possibility of identifying the short hard burst GRB 051103 with a giant flare from a soft gamma repeater in the M81 group of galaxies. Astron. Lett. 33, 19-24, DOI: 10.1134/S1063773707010021 (2007). astro-ph/0609544.

9. Mazets, E. P. et al. A giant flare from a soft gamma repeater in the andromeda galaxy (m31). The Astrophys. J. 680, 545-549, DOI: $10.1086 / 587955$ (2008).

10. Sinnott, R. W. VizieR Online Data Catalog: NGC 2000.0 (Sky Publishing, ed. Sinnott 1988). VizieR Online Data Catalog VII/118 (1997).

11. Abdollahi, S. et al. Fermi large area telescope fourth source catalog. The Astrophys. J. Suppl. Ser. 247, 33, DOI: $10.3847 / 1538-4365 / \mathrm{ab} 6 \mathrm{bcb}(2020)$.

12. Ackermann, M. et al. GeV Observations of Star-forming Galaxies with the Fermi Large Area Telescope. The Astrophys. J. 755, 164, DOI: 10.1088/0004-637X/755/2/164 (2012). 1206.1346.

13. de Ruiter, H. R., Willis, A. G. \& Arp, H. C. A Westerbork $1415 \mathrm{MHz}$ survey of background radio sources. II. Optical identifications with deep IIIa-J plates. Astron. Astrophys. Suppl. 28, 211-293 (1977).

14. Li, T. P. \& Ma, Y. Q. Analysis methods for results in gamma-ray astronomy. The Astrophys. J. 272, 317-324, DOI: 10.1086/161295 (1983).

15. Scargle, J. D. Studies in Astronomical Time Series Analysis. V. Bayesian Blocks, a New Method to Analyze Structure in Photon Counting Data. The Astrophys. J. 504, 405, DOI: 10.1086/306064 (1998).

16. Scargle, J. D., Norris, J. P., Jackson, B. \& Chiang, J. Studies in Astronomical Time Series Analysis. VI. Bayesian Block Representations. The Astrophys. J. 764, 167, DOI: 10.1088/0004-637X/764/2/167 (2013). 1207.5578.

17. Story, S. A. \& Baring, M. G. Magnetic Pair Creation Transparency in Gamma-Ray Pulsars. ApJ 790, 61, DOI: 10.1088/0004-637X/790/1/61 (2014). 1406.2767.

18. Thompson, C. \& Duncan, R. C. The soft gamma repeaters as very strongly magnetized neutron stars - I. Radiative mechanism for outbursts. Mon. Notices Royal Astron. Soc. 275, 255-300, DOI: 10.1093/mnras/275.2.255 (1995).

19. Lyutikov, M. Mass-loading of pulsar winds. Mon. Notices Royal Astron. Soc. 339, 623-632, DOI: 10.1046/j.1365-8711. 2003.06141.x (2003). astro-ph/0202344.

20. Gill, R. \& Heyl, J. S. On the trigger mechanisms for soft gamma-ray repeater giant flares. Mon. Notices Royal Astron. Soc. 407, 1926-1932, DOI: 10.1111/j.1365-2966.2010.17038.x (2010). 1002.3662.

21. Ioka, K., Razzaque, S., Kobayashi, S. \& Mészáros, P. TeV-PeV Neutrinos from Giant Flares of Magnetars and the Case of SGR 1806-20. The Astrophys. J. 633, 1013-1017, DOI: 10.1086/466514 (2005). astro-ph/0503279.

22. Nakar, E., Piran, T. \& Sari, R. Pure and Loaded Fireballs in Soft Gamma-Ray Repeater Giant Flares. The Astrophys. J. 635, 516-521, DOI: 10.1086/497296 (2005). astro-ph/0502052.

23. Gelfand, J. D. et al. A Rebrightening of the Radio Nebula Associated with the 2004 December 27 Giant Flare from SGR 1806-20. The Astrophys. J. Lett. 634, L89-L92, DOI: $10.1086 / 498643$ (2005). astro-ph/0503269.

24. Granot, J. et al. Diagnosing the Outflow from the SGR 1806-20 Giant Flare with Radio Observations. The Astrophys. J. 638, 391-396, DOI: 10.1086/497680 (2006). astro-ph/0503251.

25. Cheng, K. S. \& Wang, X. Y. The Radio Afterglow from the Giant Flare of SGR 1900+14: The Same Mechanism as Afterglows from Classic Gamma-Ray Bursts? The Astrophys. J. Lett. 593, L85-L88, DOI: 10.1086/378216 (2003). astro-ph/0307085. 
26. Hurley, K. The short gamma-ray burst - SGR giant flare connection. Adv. Space Res. 47, 1337-1340, DOI: 10.1016/j.asr. 2010.08.036 (2011).

27. Abdo, A. A. et al. Search for Gamma-ray Emission from Magnetars with the Fermi Large Area Telescope. The Astrophys. J. Lett. 725, L73-L78, DOI: 10.1088/2041-8205/725/1/L73 (2010). 1011.0091.

28. Li, J., Rea, N., Torres, D. F. \& de Oña-Wilhelmi, E. Gamma-ray Upper Limits on Magnetars with Six Years of Fermi-LAT Observations. The Astrophys. J. 835, 30, DOI: 10.3847/1538-4357/835/1/30 (2017). 1607.03778.

29. Ajello, M. et al. A Decade of Gamma-Ray Bursts Observed by Fermi-LAT: The Second GRB Catalog. The Astrophys. J. 878, 52, DOI: $10.3847 / 1538-4357 / a b 1 d 4$ e (2019).

\section{Acknowledgements}

The Fermi-LAT Collaboration acknowledges support for LAT development, operation and data analysis from NASA and DOE (United States), CEA/Irfu and IN2P3/CNRS (France), ASI and INFN (Italy), MEXT, KEK, and JAXA (Japan), and the K.A. Wallenberg Foundation, the Swedish Research Council and the National Space Board (Sweden). Science analysis support in the operations phase from INAF (Italy) and CNES (France) is also gratefully acknowledged. This work performed in part under DOE Contract DE-AC02-76SF00515.

\section{Author contributions statement}

The Fermi-LAT was designed and constructed by the Fermi-LAT Collaboration. The operation, data processing, calibration, Monte Carlo simulations of the detector and of theoretical models, and data analyses were performed by the members of the Fermi-LAT Collaboration. All Fermi-LAT collaborators that signed this paper contributed to the editing and comments to the final version of the manuscript. The contact authors for this paper are A. Berretta, N. Di Lalla, N. Omodei and F. Piron who contributed to the analysis and the writing of the manuscript and S. Razzaque who provided the interpretation and contributed to the writing of the paper.

The Fermi LAT Collaboration M. Ajello ${ }^{1}$, W. B. Atwood ${ }^{2}$, M. Axelsson ${ }^{3,4}$, L. Baldini ${ }^{5}$, G. Barbiellini6,7 ${ }^{6}$, M. G. Baring ${ }^{8}$, D. Bastieri ${ }^{9}, 10$, R. Bellazzini ${ }^{11}$, A. Berretta ${ }^{12 *}$, E. Bissaldi ${ }^{13,14}$, R. D. Blandford ${ }^{15}$, R. Bonino ${ }^{16,17}$, J. Bregeon ${ }^{18}$, P. Bruel ${ }^{19}$, R. Buehler ${ }^{20}$, E. Burns ${ }^{21,22,23}$, S. Buson ${ }^{24}$, R. A. Cameron ${ }^{15}$, P. A. Caraveo ${ }^{25}$, E. Cavazzuti ${ }^{26}$, S. Chen ${ }^{927}$, C. C. Cheung ${ }^{28}$, G. Chiaro ${ }^{25}$, S. Ciprini ${ }^{29,30}$, D. Costantin ${ }^{31}$, M. Crnogorcevic ${ }^{32}$, S. Cutini ${ }^{33}$, F. D' Ammando ${ }^{34}$, P. de la Torre Luque ${ }^{13}$, F. de Palma ${ }^{16}$, S. W. Digel ${ }^{15}$, N. Di Lalla ${ }^{15 *}$, L. Di Venere ${ }^{13,14}$, F. Fana Dirirsa ${ }^{35}$, Y. Fukazawa ${ }^{36}$, S. Funk ${ }^{37}$, P. Fusco ${ }^{13,14}$, F. Gargano ${ }^{14}$, N. Giglietto ${ }^{13,14}$, R. Gill ${ }^{38,39}$, F. Giordano ${ }^{13,14}$, M. Giroletti ${ }^{34}$, J. Granot ${ }^{39}$, D. Green ${ }^{40}$, I. A. Grenier ${ }^{41}$, S. Griffin ${ }^{22}$, S. Guiriec ${ }^{38,22}$, E. Hays ${ }^{22}$, D. Horan ${ }^{19}$, G. Jóhannesson ${ }^{42,43}$, M. Kerr ${ }^{28}$, M. Kovačević ${ }^{33}$, M. Kuss ${ }^{11}$, S. Larsson ${ }^{4,44,45}$, L. Latronico ${ }^{16}$, J. Li ${ }^{20}$, F. Longo ${ }^{6,7}$, F. Loparco ${ }^{13,14}$, M. N. Lovellette ${ }^{28}$, P. Lubrano ${ }^{33}$, S. Maldera ${ }^{16}$, A. Manfreda ${ }^{5}$, G. MartíDevesa $^{46}$, M. N. Mazziotta ${ }^{14}$, J. E. McEnery ${ }^{22,32}$, I.Mereu ${ }^{12,33}$, P. F. Michelson ${ }^{15}$, T. Mizuno ${ }^{47}$, M. E. Monzani ${ }^{15}$, A. Morselli ${ }^{29}$, I. V. Moskalenko ${ }^{15}$, M. Negro ${ }^{48,49}$, N. Omodei ${ }^{15 *}$, M. Orienti ${ }^{34}$, E. Orlando ${ }^{50,15}$, V. S. Paliya ${ }^{20}$, D. Paneque ${ }^{40}$, Z. Pei ${ }^{10}$, M. Pesce-Rollins ${ }^{11}$, F. Piron ${ }^{18 *}$, H. Poon ${ }^{36}$, T. A. Porter ${ }^{15}$, G. Principe ${ }^{34}$, J. L. Racusin ${ }^{22}$, S. Rainò ${ }^{13,14}$, R. Rando ${ }^{27,9,51}$, B. Rani ${ }^{52,22}$, S. Razzaque ${ }^{53 *}$, A. Reimer ${ }^{46,15}$, O. Reimer ${ }^{46}$, P. M. Saz Parkinson ${ }^{2,54,55}$, J. D. Scargle ${ }^{56}$, L. Scotton ${ }^{18}$, D. Serini ${ }^{13}$, C. Sgrò ${ }^{11}$, E. J. Siskind ${ }^{57}$, G. Spandre ${ }^{11}$, P. Spinelli ${ }^{13,14}$, H. Tajima ${ }^{58,15}$, M. N. Takahashi ${ }^{40}$, D. Tak ${ }^{59,22}$, D. F. Torres ${ }^{60,61}$, G. Tosti ${ }^{33,12}$, E. Troja ${ }^{22,32}$, Z. Wadiasingh ${ }^{22}$, K. Wood ${ }^{62}$, M. Yassine ${ }^{6,7}$, A. Yusafzai ${ }^{37}$, G. Zaharijas ${ }^{50,63}$

1. Department of Physics and Astronomy, Clemson University, Kinard Lab of Physics, Clemson, SC 29634-0978, USA

2. Santa Cruz Institute for Particle Physics, Department of Physics and Department of Astronomy and Astrophysics, University of California at Santa Cruz, Santa Cruz, CA 95064, USA

3. Department of Physics, Stockholm University, AlbaNova, SE-106 91 Stockholm, Sweden

4. Department of Physics, KTH Royal Institute of Technology, AlbaNova, SE-106 91 Stockholm, Sweden

5. Università di Pisa and Istituto Nazionale di Fisica Nucleare, Sezione di Pisa I-56127 Pisa, Italy

6. Istituto Nazionale di Fisica Nucleare, Sezione di Trieste, I-34127 Trieste, Italy

7. Dipartimento di Fisica, Università di Trieste, I-34127 Trieste, Italy 
8. Rice University, Department of Physics and Astronomy, MS-108, P. O. Box 1892, Houston, TX 77251, USA

9. Istituto Nazionale di Fisica Nucleare, Sezione di Padova, I-35131 Padova, Italy

10. Dipartimento di Fisica e Astronomia "G. Galilei”, Università di Padova, I-35131 Padova, Italy

11. Istituto Nazionale di Fisica Nucleare, Sezione di Pisa, I-56127 Pisa, Italy

12. Dipartimento di Fisica, Università degli Studi di Perugia, I-06123 Perugia, Italy

13. Dipartimento di Fisica "M. Merlin" dell'Università e del Politecnico di Bari, via Amendola 173, I-70126 Bari, Italy

14. Istituto Nazionale di Fisica Nucleare, Sezione di Bari, I-70126 Bari, Italy

15. W. W. Hansen Experimental Physics Laboratory, Kavli Institute for Particle Astrophysics and Cosmology, Department of Physics and SLAC National Accelerator Laboratory, Stanford University, Stanford, CA 94305, USA

16. Istituto Nazionale di Fisica Nucleare, Sezione di Torino, I-10125 Torino, Italy

17. Dipartimento di Fisica, Università degli Studi di Torino, I-10125 Torino, Italy

18. Laboratoire Univers et Particules de Montpellier, Université Montpellier, CNRS/IN2P3, F-34095 Montpellier, France

19. Laboratoire Leprince-Ringuet, École polytechnique, CNRS/IN2P3, F-91128 Palaiseau, France

20. Deutsches Elektronen Synchrotron DESY, D-15738 Zeuthen, Germany

21. Dept. of Physics and Astronomy, Louisiana State University, Baton Rouge, LA 70803, USA

22. NASA Goddard Space Flight Center, Greenbelt, MD 20771, USA

23. NASA Postdoctoral Program Fellow, USA

24. Institut für Theoretische Physik and Astrophysik, Universität Würzburg, D-97074 Würzburg, Germany

25. INAF-Istituto di Astrofisica Spaziale e Fisica Cosmica Milano, via E. Bassini 15, I-20133 Milano, Italy

26. Italian Space Agency, Via del Politecnico snc, 00133 Roma, Italy

27. Department of Physics and Astronomy, University of Padova, Vicolo Osservatorio 3, I-35122 Padova, Italy

28. Space Science Division, Naval Research Laboratory, Washington, DC 20375-5352, USA

29. Istituto Nazionale di Fisica Nucleare, Sezione di Roma "Tor Vergata", I-00133 Roma, Italy

30. Space Science Data Center - Agenzia Spaziale Italiana, Via del Politecnico, snc, I-00133, Roma, Italy

31. University of Padua, Department of Statistical Science, Via 8 Febbraio, 2, 35122 Padova

32. Department of Astronomy, University of Maryland, College Park, MD 20742, USA

33. Istituto Nazionale di Fisica Nucleare, Sezione di Perugia, I-06123 Perugia, Italy

34. INAF Istituto di Radioastronomia, I-40129 Bologna, Italy

35. Department of Physics, University of Johannesburg, PO Box 524, Auckland Park 2006, South Africa

36. Department of Physical Sciences, Hiroshima University, Higashi-Hiroshima, Hiroshima 739-8526, Japan

37. Friedrich-Alexander Universität Erlangen-Nürnberg, Erlangen Centre for Astroparticle Physics, Erwin-Rommel-Str. 1, 91058 Erlangen, Germany

38. The George Washington University, Department of Physics, 725 21st St, NW, Washington, DC 20052, USA

39. Department of Natural Sciences, Open University of Israel, 1 University Road, POB 808, Ra'anana 43537, Israel

40. Max-Planck-Institut für Physik, D-80805 München, Germany 
41. AIM, CEA, CNRS, Université Paris-Saclay, Université Paris Diderot, Sorbonne Paris Cité, F-91191 Gif-sur-Yvette, France

42. Science Institute, University of Iceland, IS-107 Reykjavik, Iceland

43. Nordita, Royal Institute of Technology and Stockholm University, Roslagstullsbacken 23, SE-106 91 Stockholm, Sweden

44. The Oskar Klein Centre for Cosmoparticle Physics, AlbaNova, SE-106 91 Stockholm, Sweden

45. School of Education, Health and Social Studies, Natural Science, Dalarna University, SE-791 88 Falun, Sweden

46. Institut für Astro- und Teilchenphysik, Leopold-Franzens-Universität Innsbruck, A-6020 Innsbruck, Austria

47. Hiroshima Astrophysical Science Center, Hiroshima University, Higashi-Hiroshima, Hiroshima 739-8526, Japan

48. Center for Research and Exploration in Space Science and Technology (CRESST) and NASA Goddard Space Flight Center, Greenbelt, MD 20771, USA

49. Department of Physics and Center for Space Sciences and Technology, University of Maryland Baltimore County, Baltimore, MD 21250, USA

50. Istituto Nazionale di Fisica Nucleare, Sezione di Trieste, and Università di Trieste, I-34127 Trieste, Italy

51. Center for Space Studies and Activities "G. Colombo", University of Padova, Via Venezia 15, I-35131 Padova, Italy

52. Korea Astronomy and Space Science Institute, 776 Daedeokdae-ro, Yuseong-gu, Daejeon 30455, Korea

53. Centre for Astro-Particle Physics (CAPP) and Department of Physics, University of Johannesburg, PO Box 524, Auckland Park 2006, South Africa

54. Department of Physics, The University of Hong Kong, Pokfulam Road, Hong Kong, China

55. Laboratory for Space Research, The University of Hong Kong, Hong Kong, China

56. Space Sciences Division, NASA Ames Research Center, Moffett Field, CA 94035-1000, USA

57. NYCB Real-Time Computing Inc., Lattingtown, NY 11560-1025, USA

58. Solar-Terrestrial Environment Laboratory, Nagoya University, Nagoya 464-8601, Japan

59. Department of Physics, University of Maryland, College Park, MD 20742, USA

60. Institute of Space Sciences (CSICIEEC), Campus UAB, Carrer de Magrans s/n, E-08193 Barcelona, Spain

61. Institució Catalana de Recerca i Estudis Avançats (ICREA), E-08010 Barcelona, Spain

62. Praxis Inc., Alexandria, VA 22303, resident at Naval Research Laboratory, Washington, DC 20375, USA

63. Center for Astrophysics and Cosmology, University of Nova Gorica, Nova Gorica, Slovenia

* Contact Author 
Methods

\section{The Large Area Telescope}

The Fermi Gamma-Ray Space Telescope was placed in a low-earth orbit on June 11, 2008. Its two scientific instruments, the Large Area Telescope (LAT) ${ }^{1}$, and the Gamma-Ray Burst Monitor (GBM) ${ }^{2}$, together provide the capability of probing emission over several decades in energy. The LAT is a pair production telescope sensitive to $\gamma$ rays in the energy range from $\sim 30 \mathrm{MeV}$ to more than $300 \mathrm{GeV}$. Whether or not a $\gamma$ ray is detected by the LAT is primarily defined by two angles: the angle $\zeta$ with respect to the spacecraft zenith, and the viewing angle $\theta$ from the LAT boresight. In the analysis performed in this paper, do not make any explicit cuts on the angle $\theta$; however, the exposure drops very quickly for $\theta$ greater than $\sim 75^{\circ}$. When we calculate the exposure and the livetime, on the other hand, we only include time intervals when the entire ROI has $\zeta<100$ deg and $\theta<$ $80 \mathrm{deg}$. The wide FOV ( $2.4 \mathrm{sr}$ at $1 \mathrm{GeV}$ ) of the LAT, its high observing efficiency (scanning the entire sky every 3 hours), its broad energy range, its large effective area, its low dead time per event $(\sim 27 \mu \mathrm{s})$, its efficient background rejection, and its good angular resolution (the $68 \%$ containment radius of the point-spread function (PSF) is $\sim 0.8^{\circ}$ at $1 \mathrm{GeV}$ ) are vastly improved in comparison with those of previous instruments. With respect to those, the LAT provides more GRB detections, higher statistics per detection, and more accurate localization. From the second LAT GRB catalog $(2 \mathrm{FLGC})^{3}$, the average detection rates for the LAT are 1.7 short GRBs and 17 long GRBs per year.

\section{Detection and localization of the LAT signal}

We perform an unbinned maximum likelihood analysis, using LAT P 8_TRANSIENT020E events within a region of interest (ROI) with a radius of $12^{\circ}$ (initially centered on the GBM final ground position ${ }^{4}$ ). We select a time interval of $10-500$ seconds after the GBM trigger time $\mathrm{T}_{0}$, which contains all the $\gamma$ rays detected by the LAT before the GRB exited its FOV. We also select the events with energies between $100 \mathrm{MeV}$ and $10 \mathrm{GeV}$, and with a zenith angle $<100^{\circ}$ to limit the contribution from the bright Earth limb. The GRB photon spectrum is modeled with a power law $\mathrm{dN} / \mathrm{dE}=\mathrm{AE} \mathrm{E}^{\Gamma}$. The main background component consists of charged particles that are mis-classified as $\gamma$ rays. It is included in the analysis using the iso_P8R2_TRANSIENT020_V6_v06.txt template. Although the contribution from the Galactic diffuse emissions is very small because of the high Galactic latitude of the GRB, it is accounted for by using the gl1_iem_v07. fits template ${ }^{1}$. No source from the LAT fourth source catalog (4FGL) is bright enough to be considered in the model of the ROI.

To localize the GRB and estimate its signal significance in the LAT, we perform a likelihood ratio test for the presence of the source at different positions ${ }^{5}$. Using the gtt smap tool, we evaluate the test statistic (TS) as twice the increment of the logarithm of the likelihood by fitting the data with and without the GRB component added to the background components in the model. The maximum value, $\mathrm{TS}_{\max }=29$, is found at a location of R.A., Dec. $=11.13^{\circ},-24.97^{\circ}(\mathrm{J} 2000)$, consistent with what was first reported by Omodei et al. ${ }^{6}$. This $\mathrm{TS}_{\max }$ value corresponds to a detection significance of $4.4 \sigma$ or $5.0 \sigma$ (one sided) if the TS distribution follows $(1 / 2) \chi_{4}^{2}$ or $(1 / 2) \chi_{2}^{2}$, respectively. As it is explained in the first LAT GRB catalog ${ }^{7}$, the two coordinates of the source are considered unknown and left free to vary in the former case (namely, 4 degrees of freedom including the two spectral parameters), while the latter case is more suitable when an external position is used as an input to the analysis (e.g., the GBM initial position here).

We compute the error contours of the source localization from the variation of the TS values around the best position, namely the TS maximum. In each pixel $i$ of the map displayed in Figure 1, we first compute the difference in $\mathrm{TS}_{\text {as }} \Delta \mathrm{TS}_{\mathrm{i}}=\mathrm{TS}_{\mathrm{max}}-\mathrm{TS}_{\mathrm{i}}$. Then, we convert it to a probability contour level assuming that the $\Delta \mathrm{TS}_{\mathrm{i}}$ is distributed as a $\chi^{2}$ with 2 degrees of freedom (the two coordinates $)^{5,7}$ :

$$
\mathrm{p}_{\mathrm{i}}=\int_{0}^{\Delta \mathrm{TS}} \chi_{2}(\mathrm{t}) \mathrm{dt} .
$$

The iso-contours containing localization probabilities of $68 \%$ and $90 \%$ are highlighted in Figure 1.

The best-fit spectral parameters obtained at the position of $\mathrm{TS}_{\max }$ are summarized in Extended Data Table 1. We also calculate the isotropic energy $\left(\mathrm{E}_{\mathrm{iso}}\right)$ and luminosity $\left(\mathrm{L}_{\text {iso }}\right)$ assuming the distance of the Sculptor galaxy of $\sim 3.5 \mathrm{Mpc}^{8}$. Finally, we use the gtsrcprob tool to compute the probability for each LAT $\gamma$ ray to be associated with the LAT-detected source. The first $\gamma$ ray exceeding a probability of $90 \%$ arrives at $\mathrm{T}_{0}+19.18 \mathrm{~s}$, with an energy of $480 \mathrm{MeV}$. A $1.3 \mathrm{GeV}$ photon is detected at $\mathrm{T}_{0}+180.22 \mathrm{~s}$, while the highest-energy $\gamma$ ray is a $1.7 \mathrm{GeV}$ photon at $\mathrm{T}_{0}+284.05 \mathrm{~s}$. All of these $\gamma$ rays belong to the SOURCE class (or to a cleaner event class), which results from a tight event classification that reduces drastically the residual background rate. Extended Data Table 2 shows all the $\gamma$ rays detected within the $12^{\circ}$ ROI with their probability to be associated with the GRB. The three $\gamma$ rays with the highest association probability $(>90 \%)$ are displayed in Figure 1 with circles of radius equal to the $68 \%$ containment radius of the LAT PSF ${ }^{9,10}$.

\footnotetext{
${ }^{1}$ Both templates are available at the Fermi Science Support Center: https: //fermi.gsfc.nasa.gov/ssc/
} 


\section{Spatial association of the high-energy emission with the Sculptor galaxy}

Four galaxies from the NGC2000 catalog ${ }^{11}$ (IC 1576, IC 1578, IC 1582 and NGC 253) are located within the ROI centered at the position of the LAT source with radius $\mathrm{r}_{99}$, and many more fainter galaxies are certainly located inside the region. Adding more galaxies from catalogs with a greater limiting magnitude (more fainter galaxies) would vastly increase the number of counterpart candidates. To take this consideration into account, we adopt the likelihood ratio (LR) method ${ }^{12}$, applied in several studies for counterpart searches in different catalogs ${ }^{13-21}$. This approach allows us to obtain and quantify the reliability of a possible $\gamma$-ray association, using the counterparts' local surface density: in this sense the LR can be used to calculate the probability that a suggested association is the true counterpart of a source. If we define $r_{\alpha, \beta}$ as the angular distance $d$ between the $\gamma$-ray localization $\alpha$ and the counterpart candidate $\beta$, scaled by the $\gamma$-ray location uncertainty (at the $68 \%$ c.l.) $r_{68}$, then it is given by

$$
r_{\alpha, \beta}=\frac{d}{r_{68}} .
$$

The probability that a counterpart $\beta$ lies at a distance $r_{\alpha, \beta}$ from the $\gamma$-ray localization $\alpha$ is distributed as a Rayleigh distribution $\left(r_{\alpha, \beta} e^{-r_{\alpha, \beta}^{2} / 2}\right.$ ), while the probability that $\beta$ is a background source that, by chance, happens to lie close to the position $\alpha$ follows a linear distribution $\left(\propto r_{\alpha, \beta}\right)$. The LR can thus be computed as:

$$
\mathrm{LR}=\frac{\mathrm{p}}{\mathrm{N}\left(\leq \mathrm{m}_{\beta}\right) \mathrm{A}},
$$

where $\mathrm{p}=e^{-r_{\alpha, \beta}^{2} / 2}, \mathrm{~N}\left(\leq \mathrm{m}_{\beta}\right)$ is the surface density of sources brighter than the counterpart candidate $\beta$ (of magnitude $\left.\mathrm{m}_{\beta}\right)$ and A is the solid angle spanned by $r_{99}$. To evaluate the surface density $\mathrm{N}\left(\leq \mathrm{m}_{\beta}\right)$, we count the galaxies brighter than the candidate $\beta$ in a region of $20^{\circ}$ around the $\gamma$-ray source. At the position of the LAT-detected source, the values of the LR for the four galaxies are LR=2.1 (IC 1576), 2.9 (IC 1578), 0.3 (IC 1582), and 60 (NGC 253). Although two NGC galaxies (IC 1576 and IC 1578) are closer to the LAT best position, the LR favors the most luminous NGC 253 (the Sculptor galaxy). To take into account the extension of the counterpart galaxy, expressed by its radial angular extent in optical $r_{\text {ext }}$, we modified equation 3 for the LR by adding in quadrature $r_{\text {ext }}$ to $r_{68}$. We can write the new equation for LR in a convenient form as:

$$
\mathrm{LR}_{\mathrm{ext}}=\frac{\mathrm{p}^{\xi}}{\mathrm{N}\left(\leq \mathrm{m}_{\beta}\right) \mathrm{A}},
$$

where the exponent $\xi$ is simply defined as:

$$
\xi=\frac{1}{1+\left(\frac{\mathrm{r}_{\mathrm{ext}}}{\mathrm{r}_{68}}\right)^{2}} .
$$

To quantify the significance of the $L R$ and $\mathrm{LR}_{\text {ext }}$ values we perform a set of $10^{5}$ simulations by randomizing the position over the sky of the LAT excess, and repeating the procedure described above. For every random position we select the maximum of the LR and $\mathrm{LR}_{\mathrm{ext}}$, that corresponds to the galaxy with greatest association probability within the ROI, and we fill a histogram with these values. The LR method can also be applied using the probability map illustrated in Figure 1. From this map we can directly evaluate $1-\mathrm{p}_{\mathrm{i}}$, with $\mathrm{p}_{\mathrm{i}}$ from equation 1 , and use it as the numerator in the LR formula. In this way, we consider the shape of the TS map and we abandon the hypothesis implicit in the Rayleigh distribution that the two spatial coordinates are are independently normally distributed. Like in the previous case, we generate $10^{5}$ observations, choosing the position of the TS map randomly on the celestial sphere. For each location, we compute the LR values for the NGC galaxies in the ROI, considering them as point-like or extended sources. The p-values quantify the potential association between the Sculptor galaxy and the LAT $\gamma$-ray source. They are defined as the number of cases where the LR is greater than that obtained for the Sculptor galaxy divided by the total number of simulated cases. They can thus be obtained from the normalized cumulative distributions, displayed in Extended Data Figure 1. The two distributions (point-like vs. extended source) are similar and yield comparable association probabilities. For the Rayleigh case, p-values range from $1.7 \times 10^{-3}$ to $2.9 \times 10^{-3}$, while using the TS map to compute the LR gives lower p-values, $3.2 \times 10^{-4}$ for point-like sources and $3.6 \times 10^{-4}$ for extended sources. Lower p-values are expected from this second analysis given the elongation of the TS map toward the Sculptor galaxy, with a smaller value for the extended case because of the large extension of the Sculptor galaxy ( $\sim 25 \operatorname{arcmin})$. Assuming that the emission detected at high energies is from a short GRB (SGRB), we can calculate the False Alarm Rate (FAR) by multiplying the $\mathrm{p}$-values by the rate of SGRBs observed by the LAT. Values range from $5.4 \times 10^{-4} \mathrm{yr}^{-1}$ to $4.7 \times 10^{-3} \mathrm{yr}^{-1}$ as summarized in the first part of Extended Data Table 3.

Both the analyses suggest strong likelihood of positional association between the Sculptor galaxy and the LAT $\gamma$-ray source. 


\section{Significance of the temporal coincidence}

From Extended Data Table 2, we can see that three $\gamma$ rays with energies $0.5,1.3$ and $1.7 \mathrm{GeV}$ are reconstructed within 1 degree of Sculptor, and they arrive within a time span of approximately $300 \mathrm{~s}$. We calculate the significance of the LAT triplet by selecting all the SOURCE events (between $100 \mathrm{MeV}$ and $300 \mathrm{GeV}$ ) received by the LAT in 12 years of data within a radius of $1^{\circ}$ from the center of the Sculptor galaxy (R.A., Dec. $=11.89^{\circ},-25.29^{\circ}, \mathrm{J} 2000$ ). The total livetime of the selected ROI is about 2.98 years. To compute the probability that three photons cluster by chance, due to statistical fluctuations of the background, in the 10-500 s interval after $\mathrm{T}_{0}$, we apply the Likelihood Ratio method described by Li \& $\mathrm{Ma}^{22}$. The maximum likelihood ratio, testing the presence of a new source, is defined as:

$$
\lambda=\left[\frac{\alpha}{1+\alpha}\left(1+\frac{\mathrm{N}_{\mathrm{B}}}{\mathrm{N}_{\mathrm{S}}}\right)\right]^{\mathrm{N}_{\mathrm{S}}}\left[\frac{1}{1+\alpha}\left(1+\frac{\mathrm{N}_{\mathrm{S}}}{\mathrm{N}_{\mathrm{B}}}\right)\right]^{\mathrm{N}_{\mathrm{B}}},
$$

where $N_{S}=3$ and $N_{B}=5361$ are respectively the number of LAT photons observed during and outside the analysis time window and $\alpha$ is the ratio between the analyzed time interval (490 s) and the total livetime (about 2.98 years). The significance $S$ of the LAT triplet signal can thus be calculated as:

$$
\mathrm{S} \approx \sqrt{-2 \ln \lambda}=5.3 \sigma
$$

corresponding to a $\mathrm{p}$-value of $8.3 \times 10^{-7}$. To estimate whether such a cluster of three events is common for the analyzed ROI, we use again the entire LAT data set of the Sculptor galaxy region to compute the time intervals $\Delta \mathrm{t}_{\mathrm{i}}$ for each triplet $i$ formed by three consecutive events:

$$
\Delta \mathrm{t}_{\mathrm{i}}=\mathrm{t}_{\mathrm{i}+2}-\mathrm{t}_{\mathrm{i}}
$$

Thus, the resulting intervals are used to create the dashed red histogram of Extended Data Figure 2, in which the blue line corresponds to the Fermi orbit period and the orange line shows the $\Delta t$ of the triplet of photons observed for the LAT-detected source. This simple analysis does not consider that the ROI periodically enters and exits the LAT FOV, potentially splitting some triplets into different time windows. To take this effect into account, we perform a second and more conservative analysis subtracting from each $\Delta t_{i}$ the duration of the time intervals during which the ROI is not observable (Bad Time Intervals, BTI). As expected, the bulk of the distribution moves toward shorter time intervals (green histogram in Extended Data Figure 2) but no significant new entries appear at the tail of the distribution. This corrected histogram is in agreement with the theoretical curve expected in case of independent events (black dashed line in Extended Data Figure 2). For a Poisson distribution of $\gamma$ ray arrival times from a steady source, indeed, the probability density $P$ to observe a triplet with time interval $\Delta t$ given the mean rate $R$ is:

$$
P(\Delta t)=R^{2} \Delta t e^{-R \Delta t}
$$

With a rate $R \approx 5.7 \times 10^{-5} \mathrm{~Hz}$, this results in a probability of $1.4 \times 10^{-4}$ for an interval shorter than $\Delta t \approx 300 \mathrm{~s}$.

We find that three events clustered in a time window shorter than the one related to the LAT source on only one occasion over 12 years (within an interval of $240 \mathrm{~s}$ starting at 2017 November 21 at 03:07:33 UTC), but the likelihood analysis of this triplet resulted in a low detection significance $\left(\mathrm{TS}_{\max }=16\right)$.

We compute the FAR for the temporal coincidence of the LAT-detected source with GRB 200415A as:

$$
\mathrm{FAR}=\pi \mathrm{R}_{\text {triplet }} \times \mathrm{R}_{\mathrm{GRB}} \times \delta \mathrm{t},[\mathrm{Hz}]
$$

where $\pi$ is the area of the circular region under consideration, $R_{\mathrm{GRB}}=3.7 \times 10^{-11} \mathrm{~s}^{-1} \mathrm{deg}^{-2}$ is the rate of SGRBs detected by the GBM, obtained from the online catalog of GBM GRBs ${ }^{23}$ and scaled by the GBM FOV, and $\delta \mathrm{t}=500 \mathrm{~s}$ is the coincidence time window after the SGRB prompt emission during which we expect a signal in the LAT data. $\mathrm{R}_{\text {triplet }}$ is the mean rate of triplets having a $\Delta t$ smaller than a fixed threshold and, for a value of $500 \mathrm{~s}$, we count only eight triplets over 2.98 years of livetime (see Extended Data Figure 2). The resulting FAR is $1.6 \times 10^{-7} \mathrm{yr}^{-1}$. Considering only events with energies greater than $480 \mathrm{MeV}$ (energy of the least-energetic photon within the cluster associated with the GRB), we find only the triplet related to the MGF and the FAR accordingly decreases to $2 \times 10^{-8} \mathrm{yr}^{-1}$.

We also apply the Bayesian Blocks (BB) algorithm ${ }^{24,25}$ to the data set with the BTI removed. We used BB to detect and characterize statistically significant variations in rates of LAT $\gamma$ rays, such as the photon time tags analyzed here. It provides optimal, maximum goodness-of-fit, segmentation of the observed time series, from among all possible partitions of the observation interval. The arrival times of the photons are binned using the BB edges, and a rate for each block is obtained by dividing its number of included photons by its width in time. The only free parameter describes the prior for the distribution of 


\footnotetext{
${ }^{2}$ The angle between the Zenith and the pointing direction of the LAT. In the standard survey observations the LAT is rocked by a specified angle toward the

northern and southern orbital poles on alternate orbits.

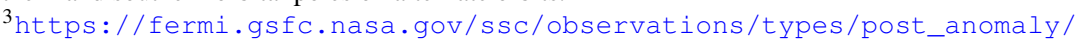

the number of blocks. Within a range suggested by calibrations based on limiting the false positive rate for single change-point detection $^{25}$, this penalty constant can be adjusted in the same spirit as with a smoothing parameter. Extended Data Figure 3 shows the results of this analysis for a selected value of the penalty constant, together with daily and weekly counts rates. We also display the weekly average exposures. Three epochs are shaded yellow, corresponding to three distinctive observing profiles. The first, at the beginning of the mission, coincides with the period in which Fermi had a $35^{\circ}$ rocking angle ${ }^{2}$. This was gradually increased until reaching $55^{\circ}$ on September 2009. Between December 2013 and July 2015, instead, Fermi spent most of its time pointing at the Galactic Center: this corresponds to the second highlighted interval, which is consequently characterized, on average, by a decrease of exposure in the direction of the Sculptor galaxy. The last highlighted period starts with the occurrence of the solar panel drive anomaly of the Fermi spacecraft ${ }^{3}$, on March 2018 and ends when a new optimized observing profile was adopted to mitigate the effect of this issue in February 2019. Spikes and dips in the exposure are the effect of occasional pointed observations (called Targets of Opportunity). However, at the time of GRB 200415A no particular features are evident in the time dependence of the accumulation of exposure. The clear spike of $\gamma$-ray rate at $\mathrm{T}_{0}$ corresponds to the cluster of the events arriving within $\approx 300 \mathrm{~s}$. In particular, there are three events in the bin with the highest rate (and a width of $810 \mathrm{~s}$ ). From simple Poisson statistics, considering the average rate of $\gamma$ rays detected from the direction of Sculptor in the remaining time history, the probability of this rate being a fluctuation is $2.3 \times 10^{-3}$.

Finally, to estimate the FAR we use a formula similar to equation 10 , with $\delta \mathrm{t}=810 \mathrm{~s}$ (the width of the time block) and $\mathrm{R}_{\text {triplet }}$ replaced by $\mathrm{R}_{\text {block}}$, namely the average detection rate of blocks exceeding a threshold of $10^{-3} \mathrm{~Hz}$. With just two such blocks in 2.98 years of total livetime (see Extended Data Figure 3), the corresponding FAR is $6.3 \times 10^{-8} \mathrm{yr}^{-1}$. These results are summarized in the second part of Extended Data Table 3.

\section{Comparison with other LAT short gamma-ray bursts}

Here we compare GRB 200415A with the population of GRBs detected by the LAT. The spectrum of GRB 200415A is typical for short bursts detected by the LAT, with a photon index $\Gamma=-1.7 \pm 0.3$ consistent with the distribution of photon indices $\Gamma_{\mathrm{EXT}}=-2.03 \pm-0.4$ (at $90 \%$ c.1.) of the 2FLGC. In that catalog, the subscript "EXT" indicates that the integration window that is used to compute the photon index is restricted to the duration of the temporally extended emission detected by the LAT, which is the most appropriate in the comparison with the photon index of GRB 200415A. The flux and fluence measured for GRB 200415A are also typical being on the low end of the distributions. What is quite peculiar about the LAT emission from GRB 200415A is its delay and duration.

The left-hand panel of Extended Data Figure 4, from the 2FLGC, shows the arrival time of the first LAT $\gamma$ ray with probability $>0.9$ of association with the GRB, which marks the beginning of the high-energy emission, as a function of the GBM $\mathrm{T}_{95}$, which marks the end of the prompt emission observed by the GBM ${ }^{26}$. For a short burst, GRB 200415A has a exceptionally delayed high-energy emission with respect to the end of the prompt phase. Two other short bursts in the 2FLGC show comparable delays: GRB 160702A was detected by Konus-Wind, INTEGRAL (SPI-ACS), Mars-Odyssey (HEND), and Swift (BAT) ${ }^{27}$. Fermi was in the South Atlantic Anomaly (SAA) at the time of the trigger, precluding a search for high-energy emission during (or immediately after) the prompt emission. Similarly, GRB 170127 was outside the FOV of the LAT, with a boresight angle of $142^{\circ}$ at the time of the GBM trigger. An Autonomous Re-point Request was issued by the GBM, and the LAT detected high-energy emission once the burst entered its FOV. GRB 200415A is the only LAT SGRB that was within the FOV at the time of trigger, and additionally its high-energy emission started much later than the end of the GBM prompt emission. The right-hand panel of Extended Data Figure 4 shows that GRB 200415A has a relatively long duration at high energies for a SGRB. Again, only the same two other SGRBs mentioned above have similar durations.

\section{GeV $\gamma$-ray flare from ultra-relativistic debris from a magnetar colliding with an outlying shell}

An MGF is a catastrophic event in the life-cycle of a magnetar, releasing a sizeable fraction of its $\sim 10^{48}$ erg magnetic energy ${ }^{28,29}$. Different trigger mechanisms have been proposed for an MGF, e.g., a rupture of the solid crust due to magnetic stress at the core-cusp boundary ${ }^{28}$, or a deformation of the magnetosphere ${ }^{30,31}$. Such a process releases a huge amount of energy within a very short period of time in a small volume near the magnetar with radius $r_{0}=10^{6} r_{0,6} \mathrm{~cm}$. This produces copious $e^{ \pm}$pairs and an optically thick fireball ${ }^{32,33}$. A qualitative description of this fireball and its evolution ${ }^{34,35}$ depends on its total luminosity $L_{0}=L_{\gamma, \text { iso }} / \xi_{\gamma} \approx 3 \times 10^{47} \xi_{\gamma,-0.5}^{-1} L_{\gamma, 47} \mathrm{erg} \mathrm{s}^{-1}$. Here $L_{\gamma, \text { iso }}=10^{47} L_{\gamma, 47} \mathrm{erg} \mathrm{s}^{-1}$ is the average isotropic-equivalent $\gamma$-ray luminosity during the prompt duration containing $90 \%$ of the fluence $T_{90}=0.141 \mathrm{~s} \mathrm{period}^{26}$, and $\xi_{\gamma}=0.3 \xi_{\gamma,-0.5}$ is the assumed fraction of the total luminosity in $\gamma$ rays, which includes the magnetic energy and kinetic energy carried by the baryons in the fireball. The initial effective temperature of the fireball is $T_{0}=\left(L_{0} / 4 \pi r_{0}^{2} c a\right)^{1 / 4} \approx 275 \xi_{\gamma,-0.5}^{-1 / 4} L_{\gamma, 47}^{1 / 4} r_{0,6}^{-1 / 2} \mathrm{keV}$; note that the luminosity is lower than that indicative of full thermalization ${ }^{26}$. Here $a=\pi^{2} k^{4} / 15 \hbar^{3} c^{3}=7.6 \times 10^{-15} \mathrm{erg} \mathrm{cm}^{-3} \mathrm{~K}^{-4}$ is the 
radiation density constant. A key finding for GRB $200415 \mathrm{~A}$ is that the total energy in the LAT emission, $E_{\mathrm{LAT}, \text { iso }}=3.6 \times 10^{45} \mathrm{erg}$, is much less than the prompt GBM energy of $1.5 \times 10^{46} \mathrm{erg}^{26}$. This implies that the fireball is ultra-relativistic and the kinetic outflow attains a terminal bulk Lorentz factor similar to a critical value obtained from the Thomson opacity argument as ${ }^{34,35}$ $\eta_{*}=\left(L_{0} \sigma_{\mathrm{T}} / 4 \pi m_{p} c^{3} r_{0}\right)^{1 / 4} \approx 140 \xi_{\gamma,-0.5}^{-1 / 4} L_{\gamma, 47}^{1 / 4} r_{0,6}^{-1 / 4}$. Here $\sigma_{\mathrm{T}}$ is the Thomson cross-section and $m_{p}$ is the mass of the proton. The total isotropic-equivalent energy of the kinetic outflow (ejecta), after decoupling from the radiation, is $E_{\mathrm{k}, \text { iso }}=3 \times 10^{46} E_{\mathrm{k}, 46.5} \mathrm{erg}$ with a bulk Lorentz factor $\Gamma_{\mathrm{ej}}=10^{2} \Gamma_{\mathrm{ej}, 2}$, where the parameters $E_{\mathrm{k}, 46.5} \sim \Gamma_{\mathrm{ej}, 2} \sim 1$. These numbers may change somewhat if the influence of field line flaring in modifying the outflow dynamics is fully taken into account. As we discuss next and in contrast to the previously modeled radio nebula from the 2004 MGF of SGR 1806-20 with an outflow velocity $\approx 0.7 \mathrm{c}^{36,37}$, this ultra-relativistic kinetic outflow is critical for our interpretation of the LAT observation.

Absent an intermediate electron acceleration site, for example a magnetic reconnection zone in the MHD wind outside the light cylinder, no significant emission is produced from the outflow before it interacts with an external shell. The external shell is naturally produced as the spindown-powered relativistic pulsar-type MHD wind emanating from the magnetar sweeps up the surrounding interstellar medium (ISM) and creates a bow shock. The radial distance of the shell is found from balancing in the rest frame of the magnetar (and of the head of the bow shock) the ram pressure of the incoming ISM with that of the MHD wind. For nominal values of the spin-down luminosity $L_{\mathrm{sd}}=10^{34} L_{\mathrm{sd}, 34} \mathrm{erg} \mathrm{s}^{-1}$, the proper motion velocity of the magnetar $v=10^{3} v_{3} \mathrm{~km} \mathrm{~s}^{-1}$ and the ISM density $n=10^{-1.6} n_{-1.6} \mathrm{~cm}^{-3}$, the radius of the bow shock is $R_{\mathrm{bs}}=\left(L_{\mathrm{sd}} / 4 \pi n m_{p} v^{2} c\right)^{1 / 2}=8 \times 10^{15} L_{\mathrm{sd}, 34}^{1 / 2} n_{-1.6}^{-1 / 2} v_{3}^{-1} \mathrm{~cm}$. The bow-shock shell has an inner part of shocked MHD wind and an outer part of shocked ISM, the two being separated by a contact discontinuity.

The observed collision time between the outflow, which propagates essentially in vacuum, and the bow-shock shell is given by $t_{\mathrm{coll}}=R_{\mathrm{bs}} / 2 \Gamma_{\mathrm{ej}}^{2} c \approx 10 \mathrm{~s}$, where we identify $t_{\mathrm{coll}}$ with the arrival time of the first photons to the observer from the head of the outflow along the line of sight. The duration of LAT emission, however, depends on the angular time scale over which emission arrives from the shocked outflow and bow-shock shell. This time scale is $t_{\theta}=R_{\mathrm{bs}} / 2 \Gamma_{\mathrm{sh}}^{2} c$, where $\Gamma_{\mathrm{sh}}$ is the bulk Lorentz factor of the forward shock propagating in the outer part of the shell with shocked-ISM (the inner part with shocked-wind offers negligible resistance). For a strong shock the density contrast between the outflow and bow-shock shell is $f \equiv n_{\mathrm{ej}} / n_{\mathrm{bs}} \approx 30$, after calculating the outflow ejecta density $n_{\mathrm{ej}}=E_{\mathrm{k}, \mathrm{iso}} / 4 \pi R_{\mathrm{bs}}^{3} m_{p} c^{2}$ and $n_{\mathrm{bs}} \approx 4 n$. As a result ${ }^{38}, \Gamma_{\mathrm{sh}}=f^{1 / 4}\left(\Gamma_{\mathrm{ej}} / 2\right)^{1 / 2} \approx 20$ and $t_{\theta} \approx 400 \mathrm{~s}$ is sufficiently long to account for the duration of the LAT emission $\approx 300 \mathrm{~s}$.

The LAT emission is produced by the shock-accelerated electrons in the material behind the forward shock that is propagating into the bow shock. The radiation efficiency $E_{\mathrm{LAT}, \text { iso }} / E_{\mathrm{k}, \text { iso }} \approx 0.1$ is typical of GRB afterglow emission. The maximum synchrotron photon energy emitted by these electrons is limited by their acceleration and cooling times to ${ }^{39}$ $E_{\mathrm{syn}, \max }=\Gamma_{\mathrm{sh}} \kappa\left(m_{e} c^{2} / \alpha_{F}\right) \approx 1.4\left(\Gamma_{\mathrm{sh}} / 20\right) \kappa \mathrm{GeV}$, where $\alpha_{F}=e^{2} / \hbar c \approx 1 / 137$ is the fine-structure constant. The factor $\kappa$ is of order unity ${ }^{40}$ and can be different for differing assumptions about electron acceleration rates and diffusion in a shock layer. Therefore, the synchrotron photon energy can explain the highest-energy LAT $\gamma$ ray observed from GRB 200415A if $\Gamma_{\text {sh }} \gtrsim 20$.

\section{References}

1. Atwood, W. B. et al. The Large Area Telescope on the Fermi Gamma-Ray Space Telescope Mission. The Astrophys. J. 697, 1071-1102, DOI: 10.1088/0004-637X/697/2/1071 (2009). 0902.1089.

2. Meegan, C. et al. The Fermi Gamma-ray Burst Monitor. The Astrophys. J. 702, 791 (2009).

3. Ajello, M. et al. A Decade of Gamma-Ray Bursts Observed by Fermi-LAT: The Second GRB Catalog. The Astrophys. J. 878, 52, DOI: $10.3847 / 1538-4357 / a b 1 d 4 e(2019)$.

4. Bissaldi, E. et al. GRB 200415A: Fermi GBM observation. GRB Coord. Netw. 27587, 1 (2020).

5. Mattox, J. R. et al. The Likelihood Analysis of EGRET Data. Astrophys. J. 461, 396, DOI: 10.1086/177068 (1996).

6. Omodei, N. et al. GRB 200415A: Fermi-LAT localization update. GRB Coord. Netw. 27597, 1 (2020).

7. Ackermann, M. et al. The First Fermi-LAT Gamma-Ray Burst Catalog. The Astrophys. J. Suppl. 209, 11, DOI: 10.1088/0067-0049/209/1/11 (2013). 1303.2908.

8. Rekola, R. et al. Distance to NGC 253 based on the planetary nebula luminosity function. Mon. Notices Royal Astron. Soc. 361, 330-336, DOI: 10.1111/j.1365-2966.2005.09166.x (2005). https://academic.oup.com/mnras/article-pdf/361/1/330/ 18658607/361-1-330.pdf.

9. Abdo, A. A. et al. The on-orbit calibration of the Fermi Large Area Telescope. Astropart. Phys. 32, 193-219, DOI: 10.1016/j.astropartphys.2009.08.002 (2009). 0904.2226.

10. Atwood, W. et al. Pass 8: Toward the Full Realization of the Fermi-LAT Scientific Potential. ArXiv e-prints arXiv:1303.3514 (2013). 1303.3514. 
11. Sinnott, R. W. VizieR Online Data Catalog: NGC 2000.0 (Sky Publishing, ed. Sinnott 1988). VizieR Online Data Catalog VII/118 (1997).

12. de Ruiter, H. R., Willis, A. G. \& Arp, H. C. A Westerbork $1415 \mathrm{MHz}$ survey of background radio sources. II. Optical identifications with deep IIIa-J plates. Astron. Astrophys. Suppl. 28, 211-293 (1977).

13. Sutherland, W. \& Saunders, W. On the likelihood ratio for source identification. Mon. Notices Royal Astron. Soc. 259, 413-420, DOI: 10.1093/mnras/259.3.413 (1992).

14. Masci, F. J. et al. VizieR Online Data Catalog: Sub-mJy radio sources complete sample (Mascit, 2001). VizieR Online Data Catalog J/PASP/113/10 (2001).

15. Ciliegi, P. et al. A deep VLA survey at $6 \mathrm{~cm}$ in the Lockman Hole. Astron. Astrophys. 398, 901-918, DOI: 10.1051/ 0004-6361:20021721 (2003). astro-ph/0211625.

16. Ackermann, M. et al. The Second Catalog of Active Galactic Nuclei Detected by the Fermi Large Area Telescope. The Astrophys. J. 743, 171, DOI: 10.1088/0004-637X/743/2/171 (2011). 1108.1420.

17. Ackermann, M. et al. The First Fermi-LAT Catalog of Sources above $10 \mathrm{GeV}$. The Astrophys. J. Suppl. 209, 34, DOI: 10.1088/0067-0049/209/2/34 (2013). 1306.6772.

18. McAlpine, K., Jarvis, M. J. \& Bonfield, D. G. Evolution of faint radio sources in the VIDEO-XMM3 field. Mon. Notices Royal Astron. Soc. 436, 1084-1095, DOI: 10.1093/mnras/stt1638 (2013). 1309.0358.

19. Ackermann, M. et al. The Third Catalog of Active Galactic Nuclei Detected by the Fermi Large Area Telescope. The Astrophys. J. 810, 14, DOI: 10.1088/0004-637X/810/1/14 (2015). 1501.06054.

20. Ajello, M. et al. The Fourth Catalog of Active Galactic Nuclei Detected by the Fermi Large Area Telescope. The Astrophys. J. 892, 105, DOI: $10.3847 / 1538-4357 / \mathrm{ab} 791 \mathrm{e}(2020)$.

21. de Menezes, R., D’Abrusco, R., Massaro, F., Gasparrini, D. \& Nemmen, R. On the Physical Association of Fermi-LAT Blazars with Their Low-energy Counterparts. The Astrophys. J. Suppl. 248, 23, DOI: 10.3847/1538-4365/ab8c4e (2020). 2004.11236.

22. Li, T. P. \& Ma, Y. Q. Analysis methods for results in gamma-ray astronomy. The Astrophys. J. 272, 317-324, DOI: 10.1086/161295 (1983).

23. von Kienlin, A. et al. The fourth fermi-GBM gamma-ray burst catalog: A decade of data. The Astrophys. J. 893, 46, DOI: 10.3847/1538-4357/ab7a18 (2020).

24. Scargle, J. D. Studies in Astronomical Time Series Analysis. V. Bayesian Blocks, a New Method to Analyze Structure in Photon Counting Data. The Astrophys. J. 504, 405, DOI: 10.1086/306064 (1998).

25. Scargle, J. D., Norris, J. P., Jackson, B. \& Chiang, J. Studies in Astronomical Time Series Analysis. VI. Bayesian Block Representations. The Astrophys. J. 764, 167, DOI: 10.1088/0004-637X/764/2/167 (2013). 1207.5578.

26. Roberts, O. J. \& the GBM and Swift Teams. Rapid Spectral Variability of a Giant Flare from an Extragalactic Magnetar. Submitted to Nature (2020).

27. Hurley, K. et al. IPN Triangulation of GRB 160702A (short/hard). GRB Coord. Netw. 19666, 1 (2016).

28. Thompson, C. \& Duncan, R. C. The soft gamma repeaters as very strongly magnetized neutron stars - I. Radiative mechanism for outbursts. Mon. Notices Royal Astron. Soc. 275, 255-300, DOI: 10.1093/mnras/275.2.255 (1995).

29. Kaspi, V. M. \& Beloborodov, A. M. Magnetars. Annu. Rev. Astron. Astrophys. 55, 261-301, DOI: 10.1146/ annurev-astro-081915-023329 (2017). 1703.00068.

30. Lyutikov, M. Mass-loading of pulsar winds. Mon. Notices Royal Astron. Soc. 339, 623-632, DOI: 10.1046/j.1365-8711. 2003.06141.x (2003). astro-ph/0202344.

31. Gill, R. \& Heyl, J. S. On the trigger mechanisms for soft gamma-ray repeater giant flares. Mon. Notices Royal Astron. Soc. 407, 1926-1932, DOI: 10.1111/j.1365-2966.2010.17038.x (2010). 1002.3662.

32. Paczynski, B. Gamma-ray bursters at cosmological distances. The Astrophys. J. Lett. 308, L43-L46, DOI: 10.1086/184740 (1986).

33. Goodman, J. Are gamma-ray bursts optically thick? The Astrophys. J. Lett. 308, L47, DOI: 10.1086/184741 (1986).

34. Ioka, K., Razzaque, S., Kobayashi, S. \& Mészáros, P. TeV-PeV Neutrinos from Giant Flares of Magnetars and the Case of SGR 1806-20. The Astrophys. J. 633, 1013-1017, DOI: 10.1086/466514 (2005). astro-ph/0503279. 
35. Nakar, E., Piran, T. \& Sari, R. Pure and Loaded Fireballs in Soft Gamma-Ray Repeater Giant Flares. The Astrophys. J. 635, 516-521, DOI: 10.1086/497296 (2005). astro-ph/0502052.

36. Gelfand, J. D. et al. A Rebrightening of the Radio Nebula Associated with the 2004 December 27 Giant Flare from SGR 1806-20. The Astrophys. J. Lett. 634, L89-L92, DOI: 10.1086/498643 (2005). astro-ph/0503269.

37. Granot, J. et al. Diagnosing the Outflow from the SGR 1806-20 Giant Flare with Radio Observations. The Astrophys. J. 638, 391-396, DOI: 10.1086/497680 (2006). astro-ph/0503251.

38. Sari, R. \& Piran, T. Hydrodynamic Timescales and Temporal Structure of Gamma-Ray Bursts. The Astrophys. J. Lett. 455, L143, DOI: 10.1086/309835 (1995). astro-ph/9508081.

39. Razzaque, S., Dermer, C. D. \& Finke, J. D. Synchrotron Radiation from Ultra-High Energy Protons and the Fermi Observations of GRB 080916C. The Open Astron. J. 3, 150-155, DOI: 10.2174/1874381101003010150 (2010). 0908.0513.

40. De Jager, O. et al. Gamma-ray observations of the crab nebula: A study of the synchro-compton spectrum. The Astrophys. J. 457, 253 (1996). 
Extended Data Table 1. Best fit parameters from the LAT unbinned likelihood analysis. All fluxes are calculated in the 100 $\mathrm{MeV}-10 \mathrm{GeV}$ energy range.

\begin{tabular}{|c|c|c|c|c|}
\hline Source & Parameter & Value & Units & T.S. \\
\hline \multirow{5}{*}{ LAT source } & Index $(\Gamma)$ & $-1.7 \pm 0.3$ & & 29 \\
\hline & Energy Flux & $(4.8 \pm 2.7) \times 10^{-9}$ & $\operatorname{erg~cm}{ }^{-2} \mathrm{~s}^{-1}$ & \\
\hline & Flux & $(4.1 \pm 2.2) \times 10^{-6}$ & $\mathrm{~cm}^{-2} \mathrm{~s}^{-1}$ & \\
\hline & $\mathrm{L}_{\text {iso }}$ & $(7.4 \pm 4.2) \times 10^{42}$ & $\operatorname{erg~s}^{-1}$ & \\
\hline & $\mathrm{E}_{\mathrm{iso}}$ & $(3.6 \pm 2.1) \times 10^{45}$ & erg & \\
\hline GalacticTemplate & Const & 1 (fixed) & & 1 \\
\hline IsotropicTemplate & Const & $1.0 \pm 0.8$ & & 3 \\
\hline
\end{tabular}


Extended Data Table 2. List of selected events, highlighting those with high probability (>90\%) to be associated with the LAT-detected source, according to the likelihood analysis. The uncertainty on the estimated $\gamma$ ray energies is of the order of 10\%. The last two columns show the angular distance to the center of NGC 253 (the Sculptor galaxy) and the 68\% containment radius of the PSF.

\begin{tabular}{crrrcrr}
$\begin{array}{c}\text { Time since } \mathbf{T}_{0} \\
(\mathrm{~s})\end{array}$ & $\begin{array}{r}\text { Energy } \\
(\mathrm{MeV})\end{array}$ & $\begin{array}{r}\text { R.A. } \\
\left({ }^{\circ}\right)\end{array}$ & $\begin{array}{r}\text { Dec } \\
\left({ }^{\circ}\right)\end{array}$ & $\begin{array}{r}\text { Prob. } \\
\text { Dist.NGC253 }\end{array}$ & $\begin{array}{r}\sigma_{68} \\
\left({ }^{\circ}\right)\end{array}$ \\
\hline $\mathbf{1 9 . 1 8}$ & $\mathbf{4 8 0}$ & $\mathbf{1 1 . 8}$ & $-\mathbf{2 5 . 0}$ & $\mathbf{0 . 9 9 0}$ & $\mathbf{0 . 3}$ & $\mathbf{1 . 0}$ \\
130.21 & 110 & 359.2 & -26.4 & 0.13 & 11.4 & 6.7 \\
135.92 & 410 & 19.9 & -25.7 & 0.13 & 7.3 & 2.3 \\
157.96 & 131 & 5.9 & -28.9 & 0.26 & 6.4 & 2.9 \\
$\mathbf{1 8 0 . 2 2}$ & $\mathbf{1 3 0 0}$ & $\mathbf{1 1 . 7}$ & $-\mathbf{2 5 . 7}$ & $\mathbf{0 . 9 8 8}$ & $\mathbf{0 . 5}$ & $\mathbf{0 . 9}$ \\
221.92 & 310 & 7.1 & -26.8 & 0.50 & 4.5 & 1.5 \\
262.17 & 350 & 16.3 & -25.9 & 0.31 & 4.1 & 1.3 \\
276.87 & 530 & 12.8 & -27.2 & 0.73 & 2.1 & 1.0 \\
$\mathbf{2 8 4 . 0 5}$ & $\mathbf{1 7 0 0}$ & $\mathbf{1 1 . 0}$ & $-\mathbf{2 5 . 0}$ & $\mathbf{0 . 9 9 9}$ & $\mathbf{0 . 9}$ & $\mathbf{0 . 4}$ \\
357.32 & 350 & 17.5 & -30.9 & 0.14 & 7.5 & 2.6 \\
471.16 & 140 & 10.1 & -21.5 & 0.75 & 4.2 & 2.8
\end{tabular}




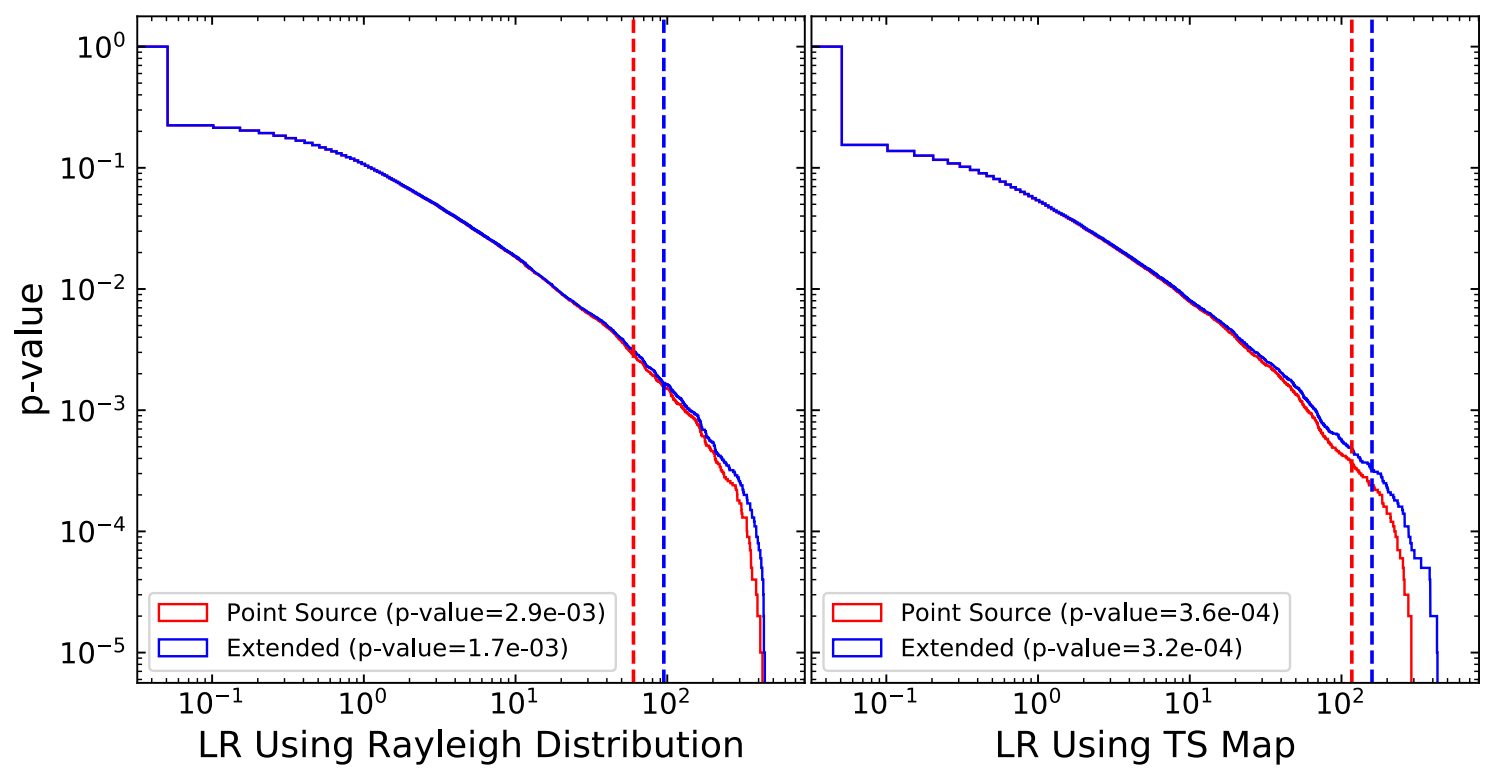

Extended Data Fig. 1. Likelihood Ratio (LR) values for $10^{5}$ simulated ROIs. Left: using the standard Rayleigh formula, right: using the TS map to compute the probability. The red distributions correspond to the point source hypothesis, while the blue distributions take into account of the galaxy extension. The step in the distributions at low LR is due to many low-LR trials occupying the first bin. The value of the LRs associated with the Sculptor galaxy are highlighted by red and blue vertical dashed lines for the two cases. 


\begin{tabular}{ccc} 
Analysis & p-value & $\begin{array}{c}\text { FAR } \\
\left(\mathrm{yr}^{-1}\right)\end{array}$ \\
\multicolumn{2}{c}{ Spatial Association with the Sculptor galaxy } \\
\hline LR (Rayleigh) & $2.9 \times 10^{-3}$ & $4.7 \times 10^{-3}$ \\
LR $_{\text {ext }}$ (Rayleigh) & $1.7 \times 10^{-3}$ & $2.9 \times 10^{-3}$ \\
LR (TS Map) & $3.6 \times 10^{-4}$ & $6.0 \times 10^{-4}$ \\
LR $_{\text {ext }}$ (TS Map) & $3.2 \times 10^{-4}$ & $5.4 \times 10^{-4}$ \\
\hline \multicolumn{3}{c}{ Temporal Association with GRB 200415A } \\
\hline Triplet Analysis & $8.3 \times 10^{-7}$ (Li \& Ma) & $1.6 \times 10^{-7}$ \\
Bayesian Blocks & $2.3 \times 10^{-3}$ (Poisson) & $6.3 \times 10^{-8}$
\end{tabular}

Extended Data Table 3. Association probability and False Alarm Rate. 


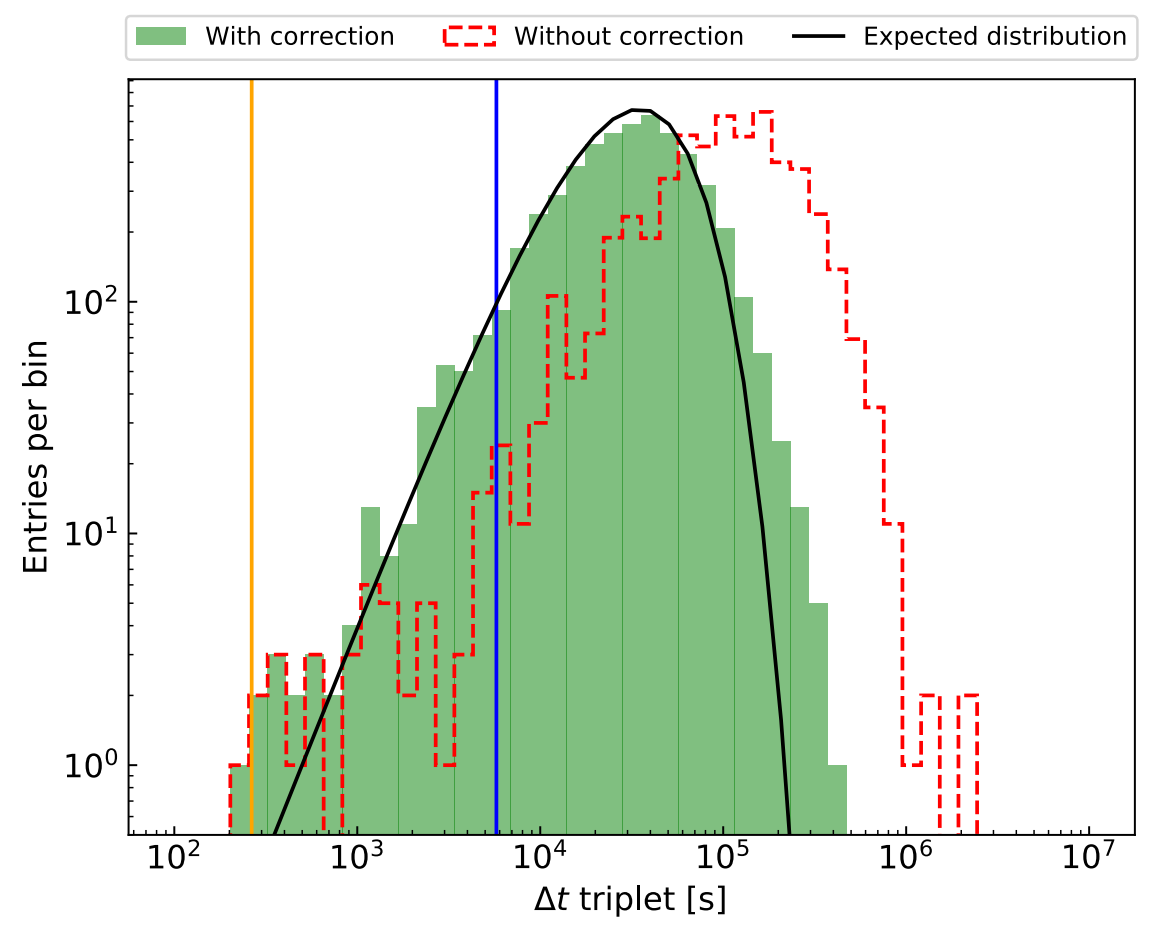

Extended Data Fig. 2. Distribution of the time intervals $\Delta \mathrm{t}$ for triplets formed by three consecutive photons with (green) and without (dashed red) taking into account the correction for the effects of the LAT orbit and FOV. The expected distribution in case of independent events is represented as a solid black line. The vertical line in blue shows the period of the Fermi orbit (5790 s), while the orange vertical line indicates $\Delta t=264.87 \mathrm{~s}$ corresponding to the photon triplet detected by the LAT after GBM detected emission from GRB 200415A. 


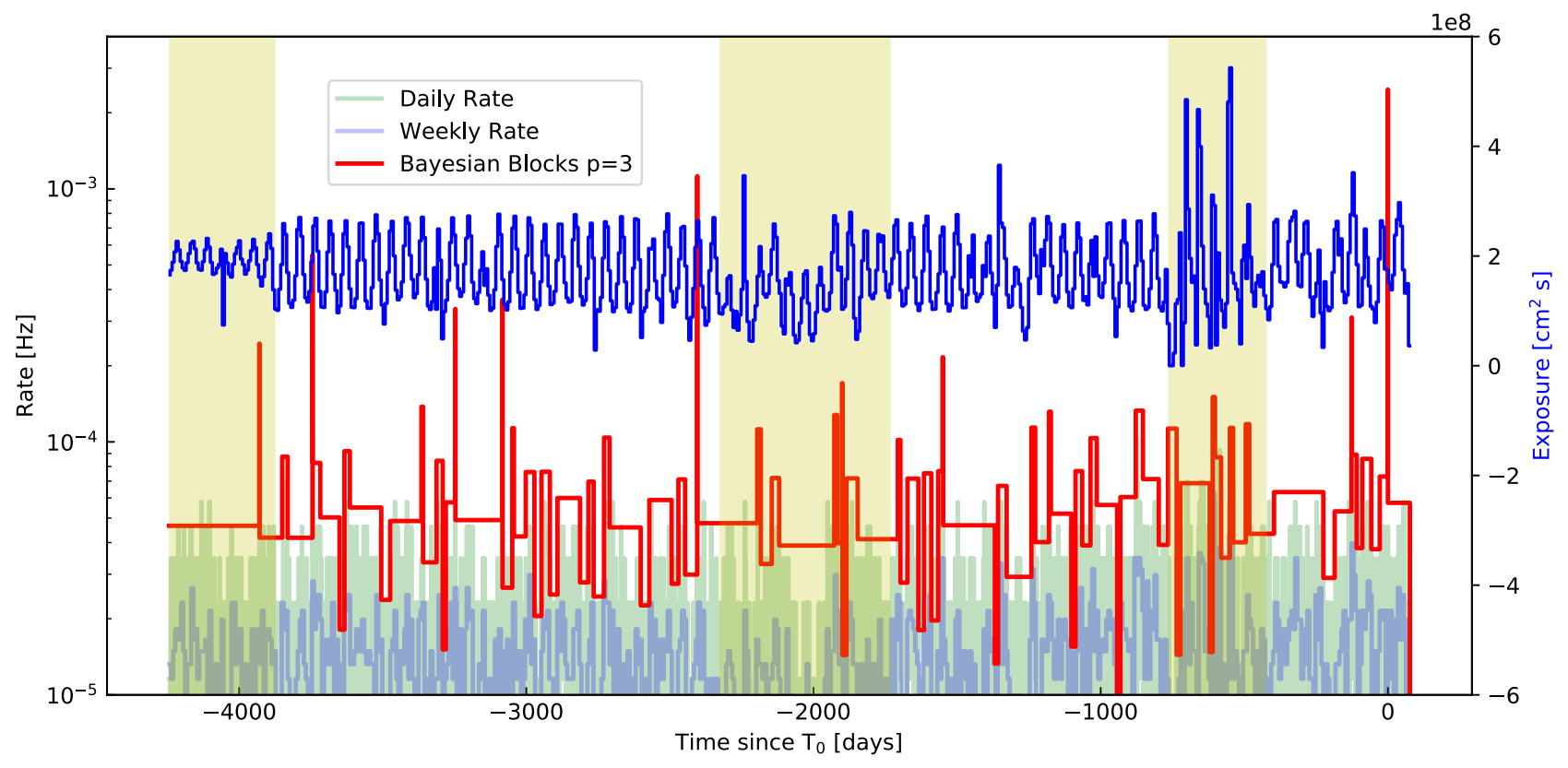

Extended Data Fig. 3. Bayesian Blocks representation of the arrival times of the $\gamma$ rays with the prior parameter $\mathrm{p}=3$. Light green and light blue are the daily and weekly count rates, while the blue curve shows the weekly-averaged exposure (between $100 \mathrm{MeV}$ and $300 \mathrm{GeV}$, assuming a power-law photon index of -2 ) for a $1^{\circ}$-radius ROI in the direction of Sculptor for the entire time of the mission. The three yellow bands highlight three characteristic observing profiles: $35^{\circ}$ rocking angle, at the beginning of the mission, an observation strategy favoring the Galactic Center region, in the middle, and, lastly, the period between the start of the solar drive anomaly and the implementation of a reoptimized survey strategy. 

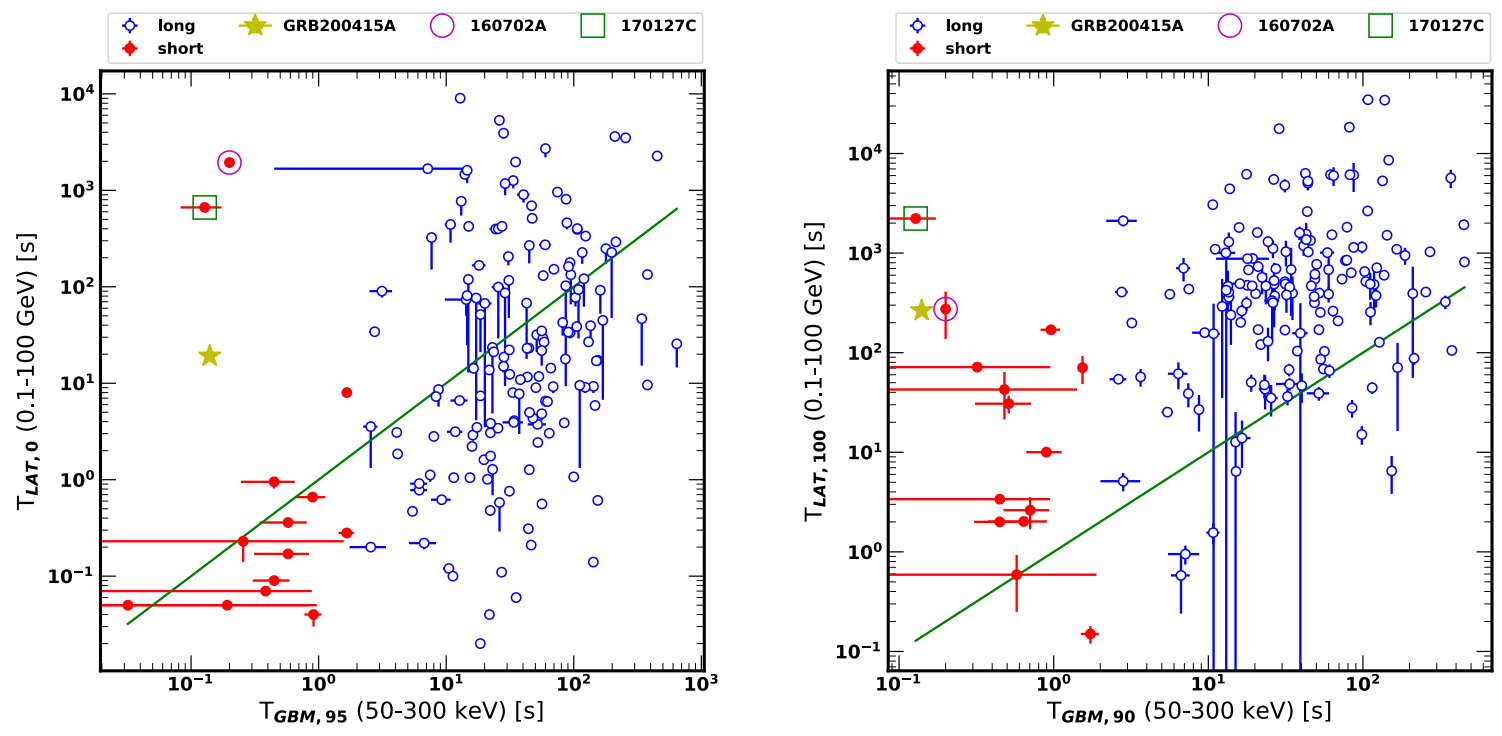

Extended Data Fig. 4. Left: onset times $\left(\mathrm{T}_{\mathrm{LAT}, 0}\right)$ in the $100 \mathrm{MeV}-100 \mathrm{GeV}$ band vs. the end of the GRB as detected by GBM in the 50-300 keV energy range ( $\left.\mathrm{T}_{\mathrm{GBM}, 95}\right)$. Right: Durations $\left(\mathrm{T}_{\mathrm{LAT}, 100}\right)$ calculated in the $100 \mathrm{MeV}-100 \mathrm{GeV}$ energy range vs. the same quantities calculated in the $50-300 \mathrm{keV}$ energy range $\left(\mathrm{T}_{\mathrm{GBM}, 90}\right)$. The solid line denotes where the two values are equal. Empty Blue and filled red circles represent long and short GRBs, respectively (data from 2FLGC ${ }^{3}$.

GRB 200415A is added and marked with a yellow star. The two SGRBs 160702A and GRB 170127C from 2FLGC, which exhibit similar durations, are highlighted with a magenta circle and green square, respectively. 